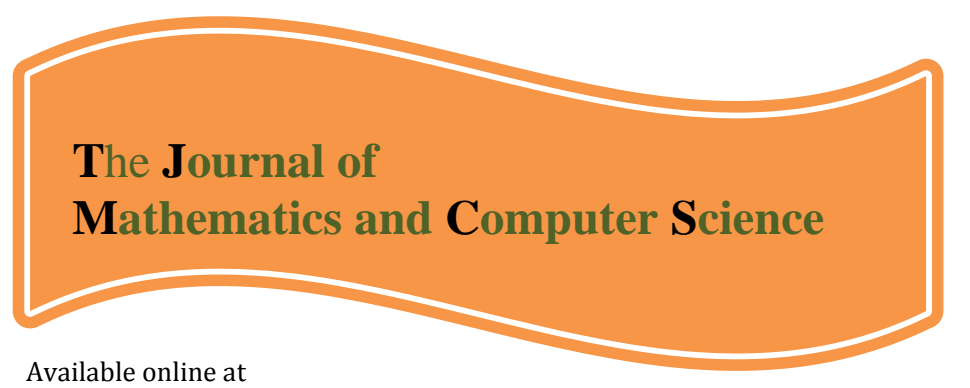

http://www.TJMCS.com

The Journal of Mathematics and Computer Science Vol .1 No.4 (2010) 247-257

\title{
Automatic Train Control based on the Multi-Agent Control of Cooperative Systems
}

\author{
Ali Siahvashi1, ${ }^{*}$, Bijan Moaveni² \\ School of Railway Engineering, Iran University of Science and Technology, Tehran, Iran, ali_siavashi@rail.iust.ac.ir \\ School of Railway Engineering, Iran University of Science and Technology, Tehran, Iran, b_moaveni@iust.ac.ir
}

Received: July 2010, Revised: September 2010

Online Publication: December 2010

\begin{abstract}
The growing traffic intensity and complexity of the railway systems as well as the demand for higher speed need to new Automatic Train Control (ATC) methods. The conventional ATC system has some problems and in recent years new ATC methods like the Decentralized ATC (D-ATC) and autonomous decentralized ATC are developed which have some advantages. In this paper, an Intelligent Decentralized ATC (ID-ATC) approach based on the Multi-Agent systems theory is developed which can provide high transportation capacity, high-safety and high-reliability. In this method we combine the Voronoi concept of cooperative systems theory with MultiAgent control theory by using of fuzzy control logic. The control algorithms are presented and by using of simulation results the effectiveness of the method is demonstrated.
\end{abstract}

Keywords: Automatic Train Control, Multi-Agent Control Systems, Cooperative systems, Fuzzy control, Voronoi Algorithm.

\footnotetext{
1,* Corresponding author: M.Sc. Student of Electrical Railway Engineering, School of Railway Engineering, Iran University of Science and Technology (IUST), Tehran, Iran, +98-21-77209041

${ }^{2}$ Assistant Professor, PhD of Control Systems Design. He is with School of Railway Engineering, Iran University of Science and Technology (IUST), Tehran, Iran, +98-21-77209041
} 


\section{Introduction}

Automatic control systems and their affects to reduce the human error problems are more attractive in recent years to control the railway transport systems due to the growing traffic intensity and complexity of these systems. Automatic Train Control (ATC) system is an automatic control algorithm to protect the trains from collision. In addition to collision avoidance, by using of the ATC system the following items can be achieved:

- Improve the performance of control and signaling systems

- Increase the safety

- $\quad$ Reduce the costs

- $\quad$ Reduce the energy consumption

According to the ATC definition, an ATC system consists of Automatic Train Protection (ATP), Automatic Train Operation (ATO) and Automatic Train Supervision (ATS). In figure 1, the fundamental structure of a typical ATC system which contains the ATP, ATO and ATS is shown. There are many kinds of ATC systems but in all of them, the ATP helps to prevent collisions through a driver's failure to observe a signal or speed restriction. The ATO provides partial or complete automatic train piloting and driverless functions and the ATS which is the basis of the train protection function and the automatic speed control devices. The ATS system, by using of the block information, specifies the speed constraints and sends them to the trains by using of Track circuit, Loop and/or Balises [1].

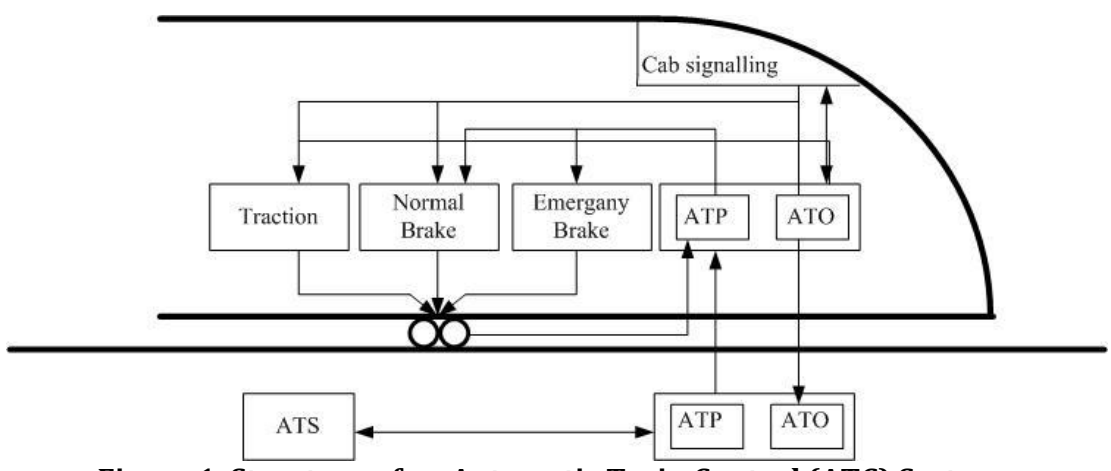

Figure 1. Structure of an Automatic Train Control (ATC) System

The recent advances in railway industries and high traffic intensity need to automatic control systems to reduce the human error problems. In last decade, some new algorithms were introduced in ATC systems which can be summarized in following titles:

- D-ATC [2]

- DS-ATC [3]

These new methods have some advantages and drawbacks in comparison with the conventional ATC algorithm. In [2], the D-ATC was introduced and they claimed that it can solve some problems and reduce complexity of the conventional analogue ATC method. Also, this method can improve the ride quality, increase the line capacity and reduce the costs by using of decentralized topology. Advantages of the D-ATC approach results in some new algorithms like algorithms of [4].

\section{Automatic Train Control based on Intelligent Decentralized Control}

In this paper, we introduce a new ATC method by using of Voronoi algorithm in cooperative systems theory. In 2004, Magnus Lindhe introduced the Voronoi algorithm by combining the Ogren effect of Navigation function and Cortes effect of Coverage Control to navigate a group of mobile robots [5]. Voronoi algorithm by using of a potential function and by finding the 
smallest path to achieve the goal presents a safe method to run the mobile robots. In this approach, a Voronoi diagram is defined as figure 2 that guarantee the collision avoidance [5].

Here, we combine the Voronoi algorithm and Mutli-Agent control systems by employing the fuzzy logic controller to present an intelligent ATC system which contains the D-ATC advantages and we call it ID-ATC. The ID-ATC can solve the problems of railway transport systems by defining the trains as agents and by using of the decentralized fuzzy controllers. In other words, each agent contains a fuzzy controller which receives the information from receptors and by analyzing them and considering the Voronoi conditions generates a control policy. In this procedure and in railway transportation system, distances between the current train and front and back trains are measured and by using of this information the decision making will be done to keep the current train in the middle point, which can guarantee the safe motion of the trains.

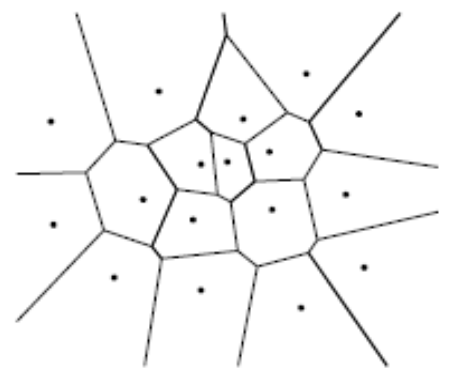

Figure 2. Voronoi diagram

\subsection{ID-ATC Algorithm}

In ID-ATC, we consider each train as an agent. Also, we consider the first train as a leader that the other agents adjust their position and consequently their velocity with the leader. In this section, we consider three trains in a line and between two stations to simplify the algorithm discussion but without any loss of generality of the problem. These three trains start the motion from the first station and stop in the last station respectively. Obviously, the number of trains is more than three in real states and to solve this problem, all trains without $1^{\text {st }}$ and last trains can be considered as middle trains.

In ID-ATC system, we propose the decentralized control structure and consequently we consider a controller for each train. The train controller plan by using of fuzzy strategy is shown in figure 3. In control procedure, each train receives the position and velocity of itself and previous and next trains. After this phase, the controller provides the appropriate control effort to control the train velocity and position. In real train control systems this control effort specifies the notch of traction system or brake system.



Figure 3. The block diagram of the control system in ID-ATC system 
In figure 3, the closed loop control system for a train is shown. This control loop contains the train dynamic model and resistance forces as running resistance and curve and gradient resistance. Also, in this closed loop control system we consider a controller to control the train velocity and position by using of the torque control strategy. According to Voronoi algorithm, this controller should keep the $2^{\text {nd }}$ train in the middle point of two other trains to obtain the safe railway transportation system. But, in this control procedure we have some constraints as

- The maximum speed of the trains should be specified.

- Trains can not go back.

- The control system of each train is independent of the others.

In ID-ATC, the $1^{\text {st }}$ train is assumed as leader agent and its desired velocity should be specified by the control center. The fuzzy control system in each train receives the velocity and position of the previous and next trains. By using of this information, the ID-ATC present the control strategy to keep the $2^{\text {nd }}$ train in the middle point between the leader and $3^{\text {rd }}$ trains. This methodology can provide safe and intelligent railway system while it improves the ride quality.

According to the above discussion about ID-ATC system and using figure 4, the following algorithm can be summarized:

1. Initializing the parameters (The parameters of the train, brake system and traction system should be specified).

2. Receiving the information of the trains (by using of sensors and Balises) and by computing the $l_{1}$ and $l_{2}$.

3. Computing the resistance forces (running resistance and effect of slope and curvature).

4. Producing the control effort by using of the Fuzzy controller.

5. Sending the control effort to the traction motor and/or brake system.

6. Return to step 1.

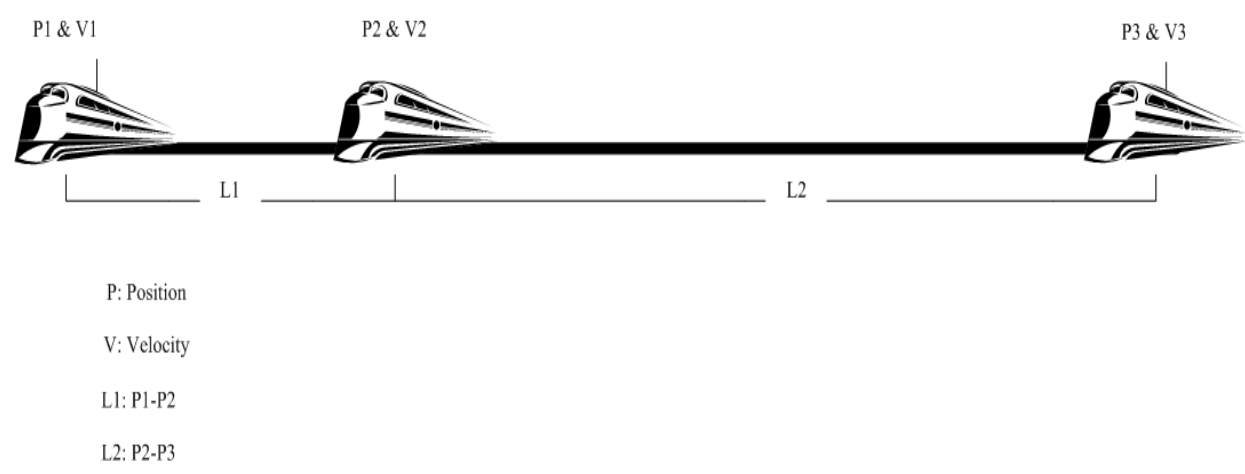

Figure 4. Three trains in a line

\subsection{Fuzzy Controller Design for ID-ATC system}

In ID-ATC system we should design a fuzzy controller for each train. So, we can categorize the fuzzy controllers in 3 groups, controller of leader train, controller of middle trains and controller of last trains. The fuzzy controller design for the leader train is trivially simple and we ignore it to present. The controller of leader train receives the desired velocity from control center and adapts its velocity by employing the fuzzy controller.

But, in the middle trains the fuzzy controller is more complex than the other controllers. In this controller, inputs are defined as:

- $l_{1}$ 
- $l_{1}-l_{2}$

- $v_{2}-v_{1}$

We consider the triangular membership functions for fuzzy controllers as figure 5 and the corresponding fuzzy rules are summarized in table 1.

Table 1. Fuzzy rules in middle trains

\begin{tabular}{|c|c|c|c|c|c|c|c|c|c|c|c|}
\hline & PS & M & PL & & PS & M & PL & & PS & M & PL \\
\hline $\mathrm{P}$ & nnn & $\mathrm{p}$ & ppp & $\mathrm{P}$ & $\mathrm{nn}$ & $\mathrm{pp}$ & pppp & $\mathrm{P}$ & $\mathrm{n}$ & ppp & ppppp \\
\hline $\mathrm{Z}$ & nnnn & $\mathrm{m}$ & $\mathrm{pp}$ & $\mathrm{Z}$ & nnn & $\mathrm{m}$ & ppp & $\mathrm{Z}$ & $\mathrm{nn}$ & $\mathrm{m}$ & pppp \\
\hline $\mathrm{N}$ & $\begin{array}{c}\text { nnnn } \\
n\end{array}$ & nnn & $\mathrm{p}$ & $\mathrm{N}$ & nnnn & $\mathrm{nn}$ & $\mathrm{pp}$ & $\mathrm{N}$ & nnn & $\mathrm{n}$ & ppp \\
\hline
\end{tabular}

where,

nnnnn: Maximum brake force (notch: \#5)

nnnn: Brake force (notch: \#4)

nnn : Brake force (notch: \#3)

$\mathrm{nn}$ : Brake force (notch: \#2)

n: Brake force (notch: \#1)

$\mathrm{m}$ : There is no force.

$\mathrm{p}$ : Traction force (notch: \#1)

pp : Traction force (notch: \#2)

ppp : Traction force (notch: \#3)

pppp : Traction force (notch: \#4)

ppppp : Maximum traction force (notch: \#5)
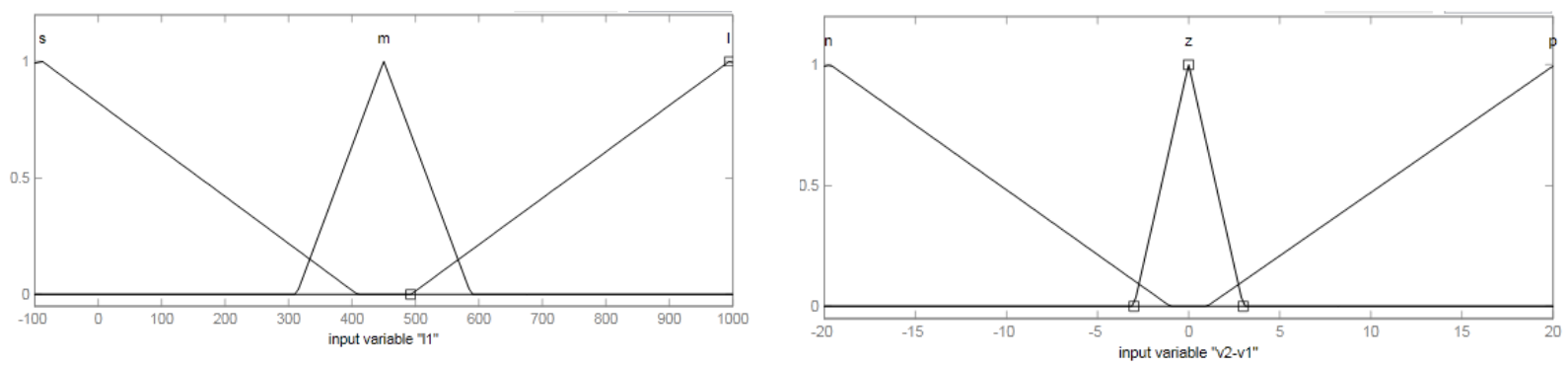

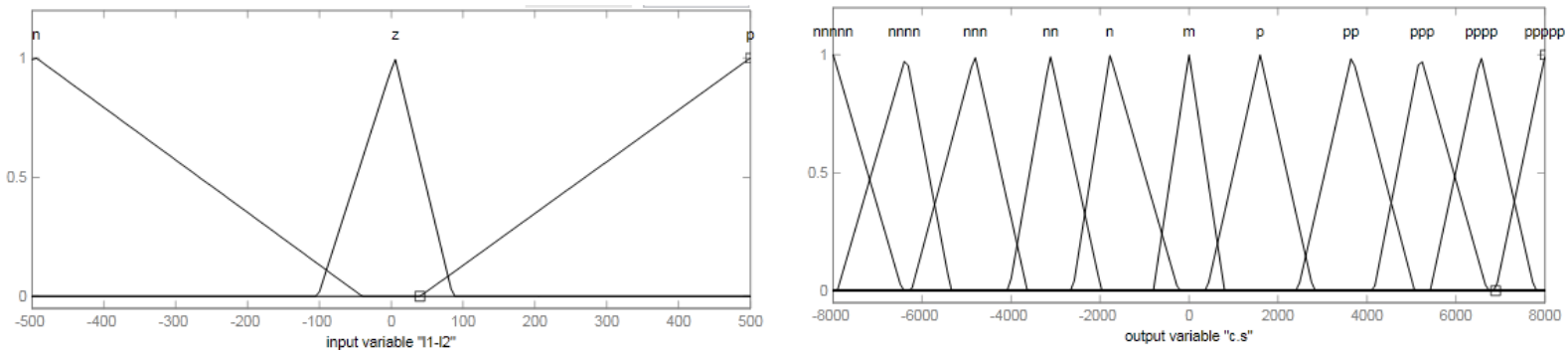

Figure 5. Membership functions of inputs and output for fuzzy controller of middle train

Also, for the last train, the fuzzy controller inputs are as follow and we employ the triangular membership functions in this controller are shown in figure 6.

- $l_{2}$

- $v_{3}-v_{2}$

The corresponding fuzzy rules in the last train are shown in table 2.

Table 2. Fuzzy rules in last train

\begin{tabular}{|c|c|c|c|}
\hline$l_{2}-v_{2}$ & $>0$ & $=0$ & $<0$ \\
\hline $\mathrm{PS}$ & $\mathrm{nnn}$ & $\mathrm{nn}$ & $\mathrm{n}$ \\
\hline $\mathrm{M}$ & $\mathrm{n}$ & $\mathrm{m}$ & $\mathrm{P}$ \\
\hline $\mathrm{PL}$ & $\mathrm{p}$ & $\mathrm{pp}$ & $\mathrm{ppp}$ \\
\hline
\end{tabular}

where,

nnn : Brake force (notch: \#3)

nn : Brake force (notch: \#2)

n: Brake force (notch: \#1)

$\mathrm{m}$ : There is no force.

$\mathrm{p}$ : Traction force (notch: \#1)

pp : Traction force (notch: \#2)

ppp : Traction force (notch: \#3)
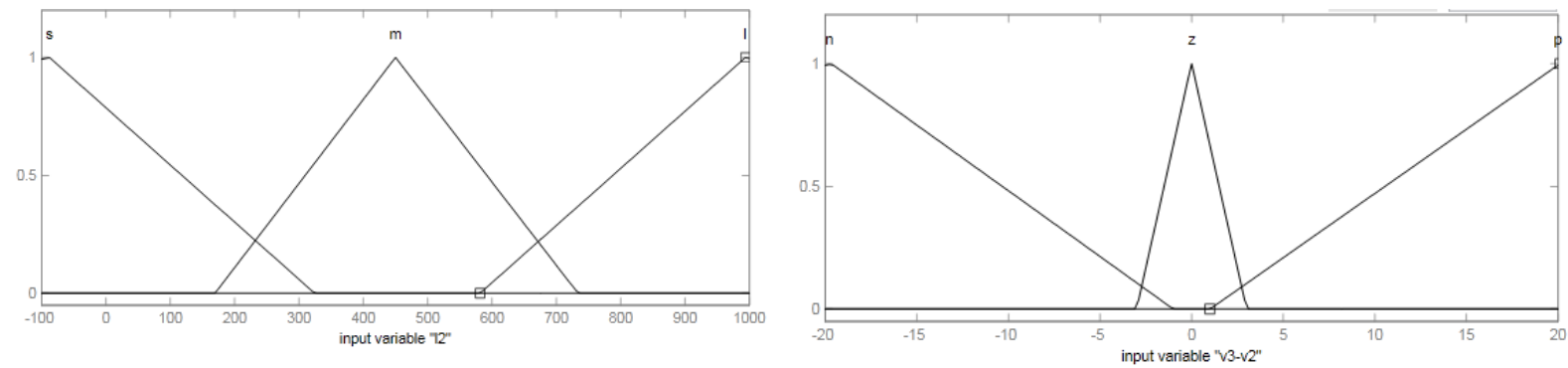


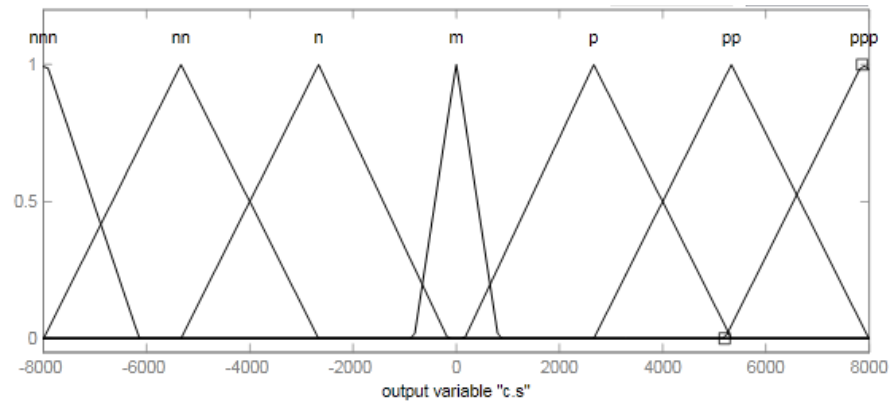

Figure 6. Membership functions of inputs and output for fuzzy controller of last train

\section{Simulation Results}

In this section we simulate the trains running by using of ID-ATC system. The train parameters, headway and initial velocity of trains are characterized according to Tehran suburban as table 3 .

Table 3. The parameters that are used is simulations

\begin{tabular}{|l|c|}
\hline Parameters of Davis Formula & $\begin{array}{c}\mathrm{a}=1.178 \\
\mathrm{~b}=0.00931 \\
\mathrm{c}=0.003648\end{array}$ \\
\hline Slope of a line (between Golbarg and Sarsabz Stations) & 400 \\
\hline Curvature (between Golbarg and Sarsabz Stations) & 400 \\
\hline Velocity of Leader Train & $55 \mathrm{~km} / \mathrm{h}$ \\
\hline Maximum velocity & $90 \mathrm{~km} / \mathrm{h}$ \\
\hline Desired Speed (1st train) & $55 \mathrm{~km} / \mathrm{h}$ \\
\hline Train weight & $40-50 \mathrm{ton}$ \\
\hline Friction Coefficient & 0.35 \\
\hline Headway & $1800 \mathrm{~m}$ \\
\hline
\end{tabular}

In figure 7 , we show the position of all three trains by using of ID-ATC system. In this procedure, the first train starts running according to the control center plan. After that, the IDATC system and the local controllers run the second and third trains respectively. In this process, the two local controllers of the second and third trains keep the second train in the middle point of the leader and the last trains, which are shown in figure 7. Whenever the control center stops the leader train in a station, the two other trains will be stopped automatically in an appropriate distance of the leader which is specified by the control center. 


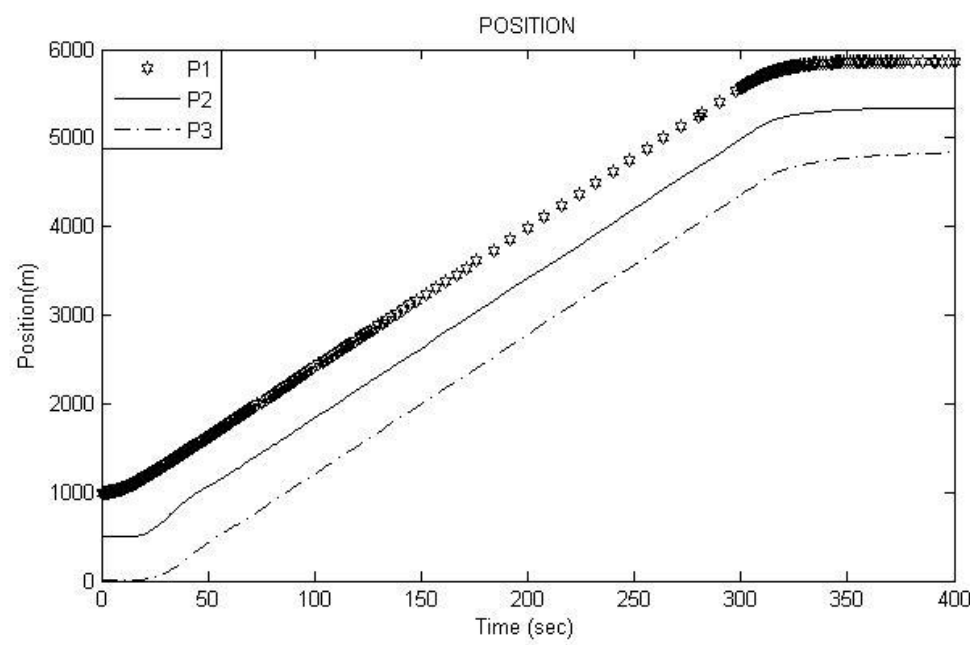

Figure 7. Trains running are controlled by ID-ATC system (Star: Position of $1^{\text {st }}$ train; Solid line: Position of $2^{\text {nd }}$ train; Dash-dot: position of $3^{\text {rd }}$ train)

In figure 8, the velocities of all three trains are shown. The velocity curves of trains show the effort of controllers to keep the trains in equal distance from each other. Also, it shows that all velocities satisfy the maximum speed limitation (Table 3 ).

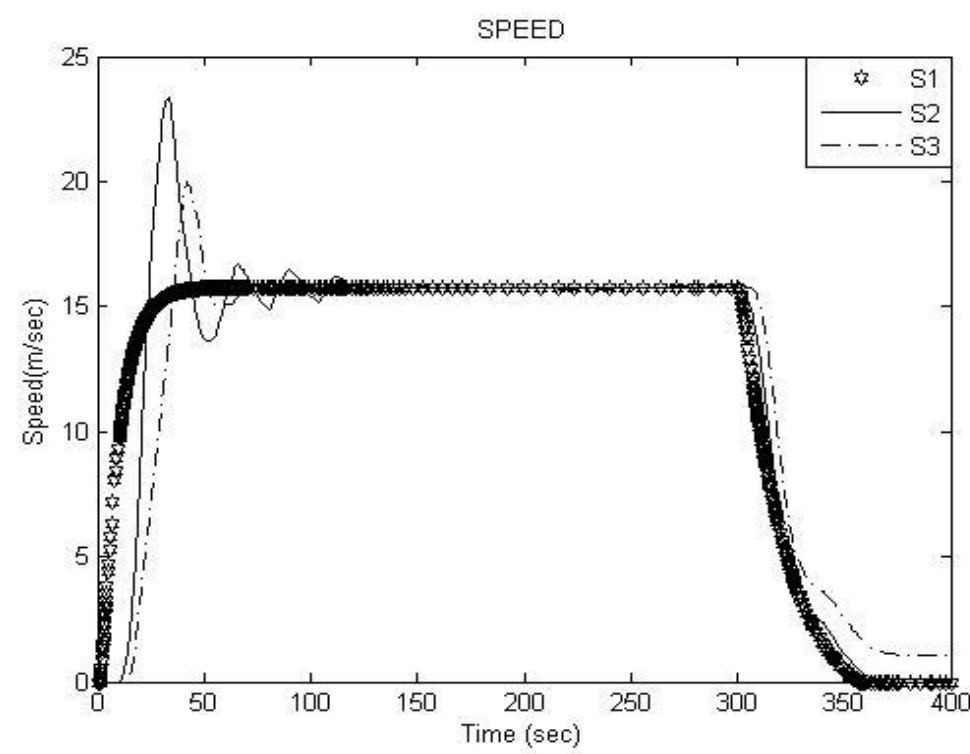

Figure 8. Trains velocity (Star: Velocity of $1^{\text {st }}$ train; Solid line: Velocity of $2^{\text {nd }}$ train; Dash-dot: Velocity of $3^{\text {rd }}$ train)

In figure 9, acceleration of the trains are shown. The acceleration curves are used to evaluate the ride quality, which should be less than $1.4^{\left(m / s^{2}\right)}$ according to UIC standards. Obviously, all trains satisfy this constraint. 


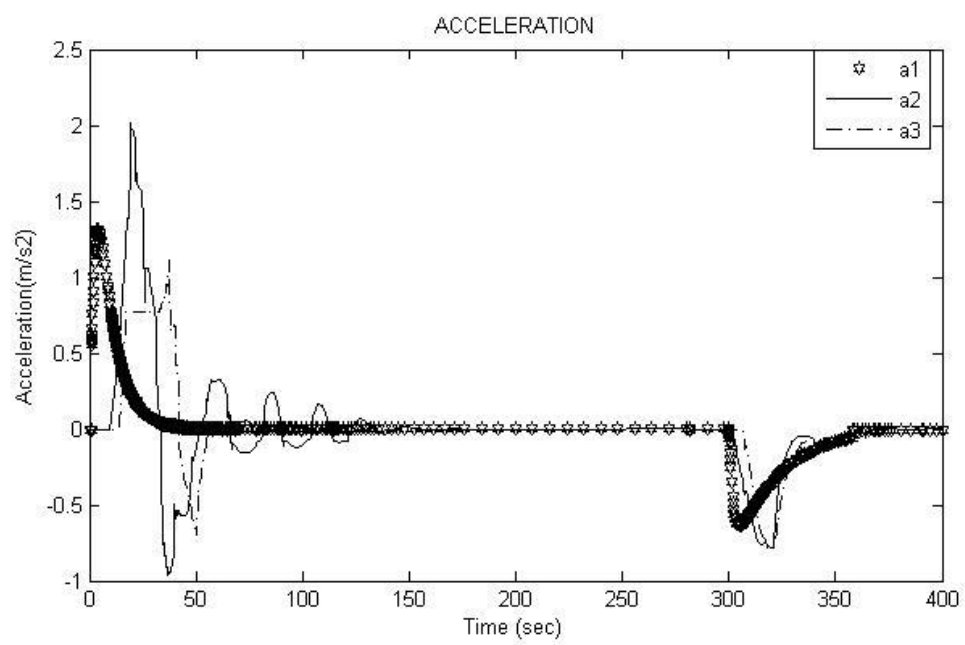

Figure 9. Trains acceleration (Star: Acceleration of $1^{\text {st }}$ train; Solid line: Acceleration of $2^{\text {nd }}$ train; Dash-dot: Acceleration of $3^{\text {rd }}$ train)

In this simulation, the desired distance between the trains is 500 meters that is characterized by headway distance in start point. To define the appropriate headway distance we should compute the maximum stopping distance to provide a safe train motion. Obviously, many parameters should be considered to compute the stopping distance [6]. Figure 10 shows the distances between the trains which are bigger than or equal to 500 meters.



Figure 10. Distance between the trains

Figure 11, shows the control efforts of $2^{\text {nd }}$ and $3^{\text {rd }}$ train which have good behavior. 




Figure 11. The control effort of $2^{\text {nd }}$ and $3^{\text {rd }}$ train (Solid line: Control effort of $2^{\text {nd }}$ Train; Doss-dotted line: Control effort of $2^{\text {nd }}$ Train)

\section{Conclusion}

In this paper, we introduce the ID-ATC system to improve the ability of conventional ATC system. This method combines the multi-agent control with cooperative systems theory and employs them to present the ID-ATC. This system has two significant advantages, decentralized structure and intelligent behavior. The decentralized structure can reduce the costs e.g. the repair and maintenance costs. Also, the intelligent behavior can provide the safety. The simulation results show the effectiveness of the method.

\section{References}

[1] Dong, H., Ning, B., Cai, B., Hou, Zh., "Automatic Train Control System Development and Simulation For High-Speed Railways", IEEE circuits and systems magazine, second quarter, Vol. 10, No. 2, pp. 6-18, 2010.

[2] Matsumoto, M., "The revolution of train control System in Japan", Proceeding of IEEE International Conference Autonomous Decentralized Systems (ISADS), pp.599-606, 2005.

[3] Matsumoto, M., Sato, M., Kitamura, S., Shigeta, T., Amiya, N., "Development of autonomous decentralized ATC system", Proceeding of the 2nd International Workshop on Autonomous Decentralized System, pp. 310 - 315, 2002.

[4] El-Kebbe, D.A., Gotz, M., "Distributed Real-Time Control of Railway Crossings Using Multi-Agent Technology", Proceeding of the International Conference on Computational Intelligence for Modeling, Control and Automation, pp. 768-772, 2005. 
[5] Lindhe, M., "A Flocking and Obstacle Avoidance Algorithm for Mobile Robots", M.Sc. thesis, KTH, Stockholm, Sweden, 2004.

[6] Yasunobu seiji, Miyamoto shoji, "A Fuzzy Control for Train Automatic Stop Control", Transaction of the Society of Instrument and Control Engineers, Vol. E.2, NO. 1, pp.1-9, 2002. 